\title{
Correction to: The Role of Continuous Cerebrospinal Fluid Pulsation Stress in the Remodeling of Artificial Vertebral Laminae: A Comparison Experiment by Li L, He Y, Chen X, Dong Y. Tissue Eng Part A 2019;25(3-4):203-213. DOI: 10.1089/ ten.tea.2018.0100
}

N the February 2019 issue of Tissue Engineering, Part A (vol. 25, no. 3-4; 203-213) the article entitled "The Role of Continuous Cerebrospinal Fluid Pulsation Stress in the Remodeling of Artificial Vertebral Laminae: A Comparison Experiment" by Li et al. requires correction.

A grant in the Acknowledgment section was incorrectly listed. The original Acknowledgment read:

\section{Acknowledgments}

"The study was supported by National Natural Science Foundation of China (H0606); by Natural Science Foundation of Minhang District, Shanghai (2014MHZ004); and by the Foundation of Shanghai Municipal Commission of Health and Family Planning (20134305).”

The Acknowledgment should have read:

\section{Acknowledgment}

The study was supported by National Natural Science Foundation of China (Grant No. 81672179); by Natural Science Foundation of Minhang District, Shanghai (2014MHZ004); and by the Foundation of Shanghai Municipal Commission of Health and Family Planning (20134305).

The online version of the article has been corrected to reflect this. The authors apologize for this error. 